\title{
The coefficient of technical readiness as an indicator of the effectiveness of the strategy of technical operation of lifting and transport equipment of terminals
}

\author{
Igor Zub ${ }^{1, *}$, Yuri Yezhov ${ }^{1}$, and Nikolai Stenin ${ }^{1}$ \\ ${ }^{1}$ Admiral Makarov State University of Maritime and Inland Shipping, Russia
}

\begin{abstract}
Technical operation of lifting and transport equipment is one of the key problems of ports and terminals. The functions of technical operation include maintenance and repair of lifting and transport equipment. The imperfection of the maintenance and repair system leads to downtime of lifting and transport equipment, which affects the cost of loading and unloading operations. The main requirements for technical operation are to ensure the quality and speed of loading and unloading operations with minimal operating costs. One of the indicators for assessing the quality of technical operation is the coefficient of technical readiness, which shows that the probability that the lifting and transport equipment will be in a working state at any time, except for periods in which operation is not provided. These periods include the time when the lifting and transport equipment is being maintained and repaired. Technical operation strategy is selected based on the technical readiness coefficient.
\end{abstract}

\section{Introduction}

Technical operation (TO) of lifting and transport equipment (LTE) is one of the key problems of ports and terminals. The functions of technical operation include maintenance and repair (MR) of the LTE. The imperfection of the MR system leads to downtime of the LTE, which affects the cost of loading and unloading operations (LUO). The main requirements for technical operation are to ensure the quality and speed of loading and unloading operations with minimal operating costs. Currently, there is no single approach to the technical operation of port lifting and transport equipment. This situation can be explained by the following reasons:

1) outdated rules of technical operation of lifting and transport equipment (RD 31.01.0204) $[1,2]$;

2) the variety of the used equipment used;

3) the lack of modern scientific studies of the processes of technical operation of the port LTE.

\footnotetext{
*Corresponding author: zubiv@gumrf.ru
} 
TO is a complex organizational and technical system, which is a subsystem of a stevedoring company (StC), and has an important direction in the strategic development plan. When creating a strategic plan for the development of the StC, a technical policy strategy is worked out that ensures competitiveness. Currently, there may be several StCs located on the territory of the port, which are separate enterprises and compete with each other. The main areas of the technical policy of the StC include:

- selection of LTE (manufacturer, model range, new or from the secondary market);

- service life of the LTE (operation of the LTE up to: a certain operating time of engine hours; maximum technical condition; a certain calendar operating time);

- strategy of TO (carrying out maintenance (M): by the schedule, in the certain operating time, the number of processed goods; carrying out repair: $(\mathrm{R})$ according to the schedule, to technical condition, in the case of failure);

- selection of repair personnel (full-time or signing a contract with an outsourcing organization).

\section{Research methods}

On the basis of the analysis and synthesis of scientific research, the analysis of the possibility of using the technical readiness coefficient $\left(\mathrm{K}_{\mathrm{TR}}\right)$ as an indicator of the effectiveness of the chosen TO strategy is carried out. Based on the generalization of the results obtained, it was concluded that the $\mathrm{K}_{\mathrm{TR}}$ of LTE can serve not only as an indicator of the effectiveness of the TO strategy, but also influence its choice.

\section{Results}

The TO strategy should be optimal in terms of the indicator that characterizes the quality of operation of the LTE. When choosing the optimal TO strategy, the possibility of adjusting the rules of technical operation (RTO) is taken into account. The indicators of the quality of operation of the LTE include:

- coefficient of technical readiness of $\mathrm{K}_{\mathrm{TR}}$;

- the probability of completing the task (operational readiness coefficient) $\mathrm{K}_{\mathrm{op}}$;

- coefficient of technical use of $\mathrm{K}_{\mathrm{TU}}$;

- average profit per unit of calendar time S;

- average costs per unit of operating time C.

To select the optimal strategy, it is proposed to use the minimax method, which consists in first finding the worst (in terms of quality) among the distribution functions that characterize the operation of a technical object, and then determining the optimal control under these conditions [3].

The TO strategy of LTE is formed on the basis of:

- available operational experience (failure statistics, operating time to failure);

- the coefficient of technical readiness of $\mathrm{K}_{\mathrm{TR}}$;

- the coefficient of technical use of $\mathrm{K}_{\mathrm{TU}}$.

The trend in the development of modern $\mathrm{StC}$ terminals involves reducing the downtime of vehicles (V) on the LUO. This indicator can be achieved by maintaining and ensuring the operability of the LTE. The processing speed of the vehicle is affected not only by a working LTE, but also by the LUO technology, the availability of a reserve and the use of modern high-performance LTE. Not all ports or StCs located on their territory can have a reserve LTE or perform technical re-equipment of the terminal due to the high cost of modern LTE. 
When deciding whether to reduce the LUO time by changing the technology, it is necessary to address the question of economic feasibility. In this case, events can develop in two ways:

1) The change of the LUO technology does not entail a change in the LTE fleet;

2) The change of the LUO technology is only possible with a complete or partial replacement of LTE fleet.

Both in the first and in the second variants, the main indicators are: cargo traffic, the productivity of the LTE fleet, the development strategy of the StC.

The "efficiency-cost" indicator can serve as an express analysis of the feasibility of introducing new equipment [4]:

$$
\mathrm{K}_{\mathrm{ef}-\text {-cost }}=\mathrm{P}_{\mathrm{LTE}} / \mathrm{C} \text {, }
$$

where $\mathrm{P}_{\mathrm{LTE}}$ - is the performance of the new LTE, C - is the cost of the new LTE and operating costs.

Next, the unit cost of a unit of productivity (TEU/rub., $\mathrm{m}^{3} / \mathrm{rub}$, ton/rub., etc.) of the LTE is determined:

$$
\mathrm{k}_{\text {unit cost }}=1 / \mathrm{K}_{\text {ef.-cost }}
$$

whence it follows that:

$$
\mathrm{k}_{\text {unit cost }}=\mathrm{C} / \mathrm{P}_{\mathrm{LTE}}
$$

When comparing indicators $K_{\text {ef.-cost.1 }}$ - for the new LTE and $K_{\text {ef.-cost.2 }}-$ for the operated LTE, it is advisable to replace the LTE if the inequality is met:

$$
\mathrm{K}_{\text {ef.-cost.2 }}-\mathrm{K}_{\text {ef.-cost.1 }}>0 \text {, }
$$

or

$$
\mathrm{k}_{\text {unit cost. } 2}-\mathrm{k}_{\text {unit cost. } 1}<0 .
$$

If equation (5) is less than zero for any values $k_{\text {unit cost.1 }}$ and $k_{\text {unit cost.2, }}$, then it is economically feasible to introduce a new LTE.

The operation of the LTE is associated with a reduction in its resource. The reduction of the resource is due to the aging processes of the metal, which results in the formation of cracks in the metal structures, which lead to changes in the operating parameters, as a result of which a failure or accident may occur. Corrosion and friction processes occurring in the friction pairs lead to wear and destruction of parts of aggregates and mechanisms. To ensure the operational state of the LTE, regardless of the financial state of the StC, a TO system is being developed.

The main task of the TO of LTE is to maintain and / or restore a working, and in some cases, serviceable technical condition, as well as to monitor compliance with the current norms and rules during the operation of the LTE. The efficiency of the LTE is ensured both by the prevention and timely elimination of failures, and by the organization of diagnostics of nodes and mechanisms. Based on the analysis of the obtained diagnostic results, the reliability of LTE is predicted. The availability of statistical data and diagnostic results allows calculating the average operating time for failure of individual units and mechanisms. To prevent an emergency stop of the LTE, preventive technical measures are carried out, spare parts are ordered, which reduces the time spent by LTE under repair. Based on statistical data and diagnostic results, routine maintenance and repair works (MR) are planned, which allows forming a staff of repair personnel and effectively manage them, regardless of their organizational form (full-time or outsourced organization).

The implementation of MR schedules does not always give the desired result - the absence of failures. The search for optimal management of TO has identified various 
strategies, which are based on different principles for restoring and sustaining the efficiency of LTE of organizational and technical measures. The recovery time $\left(\mathrm{t}_{\text {recovery }}\right)$ of LTE depends on the development and implementation of organizational and technical measures, which is one of the criteria for evaluating the TO system and is subject to optimization.

The most common system of organizational and technical measures to maintain and restore the working condition of technical facilities is the system of planned preventive repairs (PPR), within which a system of MR is being developed. In the PPR system, each technical object undergoes a repair cycle during operation through certain periods of time, which can be expressed in calendar terms of inter-repair periods or depending on the operating time of the engine hours. The PPR system implies a rational staffing table and distribution of employees of the repair service, the order of spare parts and consumables, tools, auxiliary equipment used for the repair of LTE components and mechanisms. For each planned repair, a technological card of the repair work is developed, and the start and end time of the repair is planned.

At the same time, the PPR system has a number of disadvantages:

- equipment repairs are carried out without the actual need;

- the need to take the equipment out of operation, followed by full or partial disassembly to carry out defect detection of parts;

- replacement of components and parts with a large residual resource with new ones.

Analyzing the shortcomings of the PPR, a number of authors [5, 6, 7, 8, 9, 10, 11, 12, 13] consider that with the transition to a market economy, the PPR system has lost its relevance, and the system of repairs for technical condition is more effective. Depending on the operating conditions, the criteria for carrying out repairs on the technical condition are selected. These criteria include: control of parameters, when the equipment is taken out for repair when the parameters reach the critical zone, and reliability, when the criteria is the reliability of the equipment. When controlling the parameters, the frequency of repairs is set depending on the technical condition of the parts, the process of failure of which is gradual, relative to the limits of their tolerances. When monitoring the reliability of equipment, the volume and frequency of repairs are set depending on the level of reliability of the equipment $[4,14,15]$.

In addition to these two repair strategies, there is a strategy for repairing equipment after its failure $[14,15,16,17]$.

Any strategy must be effective and have a feasibility study. One of the parameters that can be used to evaluate the effectiveness of the LTE is the coefficient of technical readiness $\left(\mathrm{K}_{\mathrm{TR}}\right)$ [3].

LTE has different technical conditions, but it can only be in one condition at the same time $i=1,2, \ldots, n$ out of the many possible ones $(i \in E)$. At the initial moment of time $t=0$, the LTE is in a working or operable condition. During operation $t$, the LTE changes from condition $\mathrm{i}$ to $\mathrm{j}$. In the condition $\mathrm{j}$, the LTE stays for a random time before moving to the next condition. The probability of such a transition will have the form $[5,18]$ :

$$
P_{i j}(t)=P_{i j} F_{i j}(t)
$$

where $F_{i j}(t)$ - function of distribution of transition time from the condition $i$ to $j$.

For finite processes with communicating conditions, we obtain the following equation:

$$
\lim P_{i j}(t)=\frac{\mu_{j}}{l i j},
$$

where $l_{i j}$ - average time to the first hit of the LTE from the condition $i$ to $j, \mu_{j}$ - average time of staying in the condition $\mathrm{j}$. 
Equation (7) represents the utilization coefficient $\left(K_{u}\right)$ of the LTE in the condition $j$, if this is the objective condition of operation. In this case $\left(\mu_{\mathrm{j}} / \mathrm{l}_{\mathrm{ij}}\right)$ - is the objective function of the technical operation process and this function can be considered as the $K_{u}$, and the maximum of the function as the criteria for the optimality of technical operation. At the same time, the $K_{u}$ shows the ratio of the time when the LTE is in working or operable condition for a given period of operation, to the time of working or operable condition and all downtime for maintenance and repairs for the considered period of operation. In the absence of cargo traffic, the LTE can be taken out for preventive works, but this will immediately reduce the $\mathrm{K}_{\mathrm{u}}$, therefore, the $\mathrm{K}_{\mathrm{u}}$ at full load of the terminal can serve as an additional indicator of the efficiency of technical operation.

Two TO strategies are considered in work [16]: the $C_{a}$ strategy - only emergency repairs are carried out, and the $\mathrm{C}_{0}$ strategy - periodic repairs are carried out. When the LTE is operating without failure for the specified repair period $\left(\mathrm{t}_{\mathrm{мрп}}\right)$ then preventive repair is carried out. The criteria for selecting the strategy were adopted:

- minimum cost of repair work for a certain operational period $\mathrm{R}(\mathrm{t}) \rightarrow \mathrm{min}$;

- statistics of failures of LTE units and mechanisms;

- coefficient of technical readiness of LTE $\mathrm{K}_{\mathrm{TR}} \rightarrow$ max.

When performing mathematical calculations, the authors came to the conclusion that the repair costs during the time between failures are less than in the production of preventive repairs, therefore, according to the authors, it is not economically profitable to carry out preventive repairs.

In real operating conditions, it is characteristic that the time-to-failure distribution function $F_{a}(t)$ and the preventive repair distribution function $F_{p}(t)$ do not coincide:

$$
\mathrm{F}_{\mathrm{a}}(\mathrm{t}) \neq \mathrm{F}_{\mathrm{p}}(\mathrm{t})
$$

The inequality (8) allows choosing the optimal strategy from the strategies $C_{0}$ and $C_{a}$. Continuing the research on the choice of the TO strategy, the $\mathrm{R}(\mathrm{t})$ and $\mathrm{K}_{\mathrm{TR}}$ functions were presented in the form of [19]:

$$
\begin{gathered}
R(t)=\frac{c_{a} F_{p}(t)+c_{p} \bar{F}_{a}(t)}{F_{p}(t) \int_{0}^{t} \bar{F}_{a}(t) d t+\bar{F}_{a}(t) \int_{0}^{t} \bar{F}_{p}(t) d t}, \\
\mathrm{~K}_{\mathrm{TR}}(\mathrm{t})=\frac{1}{\mathrm{R}_{1}(\mathrm{t})+1},
\end{gathered}
$$

where $c_{a}$ and $c_{p}$ - average costs for emergency and preventive repair, respectively; $R_{1}(t)-$ cost function, when replaced in the function $R(t) c_{a}$ and $c_{p}$ to $T_{a}$ and $T_{p}$ - the average time spent on emergency and preventive repair, respectively. It follows from (10) that the maximum $K_{T R}$ is reached at the minimum point of the function $R_{1}(t)$.

When distributing the operating time after emergency and preventive repairs according to exponential laws:

$$
F_{a}(t)=1-e^{-a t}, F_{p}(t)=1-e^{p t}, a, p>0,
$$

and when the following inequality is satisfied:

$$
\mathrm{k}<1 /(1+\Pi), \mathrm{c}=\mathrm{c}_{\mathrm{p}} / \mathrm{c}_{\mathrm{a}}, \mathrm{k}=\mathrm{p} / \mathrm{a},
$$

in accordance with economic indicators, the $C_{0}$ strategy is optimal compared to the $C_{a}$ strategy. But in case when $p=a, F_{a}(t)=F_{p}(t)$, carrying out preventive repairs will be impractical, and the optimal strategy in this case will be $\mathrm{C}_{\mathrm{a}}$. 
We consider in the work [20] the problem of finding the lower confidence bound of the $\mathrm{K}_{\mathrm{TR}}$ for systems with recoverable elements by a method based on the use of confidence bounds for the reliability parameters of individual elements with the same confidence coefficient. The essence of this method is that this coefficient for a single element passes to the entire system and a change in the technical condition of one part can lead to changes in the technical condition of the unit or mechanism.

The organization of preventive works for MR in the TO system allows maintaining the $\mathrm{K}_{\mathrm{TR}}$ at the optimal level, which can be set: $\mathrm{K}_{\mathrm{TR}} \rightarrow$ opt., the evaluation of the efficiency of the operation of the StC terminal's of LTE fleet can be carried out using the $\mathrm{K}_{\mathrm{TR}}$, which is a comprehensive indicator of the reliability of a technical object and is determined by the equation:

$$
\mathrm{K}_{\mathrm{TR}}=\frac{\mathrm{T}_{\mathrm{p}}}{\mathrm{T}_{\mathrm{p}}+\mathrm{T}_{\mathrm{BP}}},
$$

where $T_{p}$ and $T_{B P}$ - accordingly, the execution time of LUO of LTE and restoration of the technical object's efficiency, hours:

$$
\mathrm{T}_{\mathrm{BP}}=\mathrm{T}_{\mathrm{M}}+\mathrm{T}_{\mathrm{sc} \cdot \mathrm{m}}+\mathrm{T}_{\mathrm{em} \cdot \mathrm{r}}
$$

where $T_{M}$ - time spent on maintenance, $T_{s c \cdot m}$ - the time spent on scheduled maintenance, $\mathrm{T}_{\text {em.r }}$ - time spent on emergency repairs.

The concept of interval $K_{T R}$ is introduced to assess the reliability of LTE at the time interval required for performing LUO [17]. In this case, the $\mathrm{K}_{\mathrm{TR}}$ is considered as an indicator of the performance of the LTE of its functional purpose with a high probability. When determining the $\mathrm{K}_{\mathrm{TR}}$, the equation (13) is presented as:

$$
\frac{\mathrm{T}_{\mathrm{BP}}}{\mathrm{T}_{\mathrm{p}}}=\frac{1-\mathrm{K}_{\mathrm{TR}}}{\mathrm{K}_{\mathrm{TR}}} .
$$

From the equation (15), it is possible to determine the ratio of the duration of $\mathrm{T}_{\mathrm{BP}}$ and $\mathrm{T}_{\mathrm{p}}$, which is assigned at the $\mathrm{K}_{\mathrm{TR}}$ required to perform the technological operation.

If we consider LTE as a system with independent recovery of elements, then the lower confidence bound for $\mathrm{K}_{\mathrm{TR}}$ is constructed by the method of confidence bounds for the reliability parameters of individual elements of the LTE with the same confidence coefficient. The confidence factor for LTE is maintained when transition from individual elements to the LTE as a whole.

Since the system availability factor $\mathrm{K}_{\mathrm{TR}}(\mathrm{u})=\exp [\mathrm{f}(\mathrm{u})]$, we can also calculate the lower $\gamma$ - confidence bound for the coefficient of system readiness [19]:

$$
\mathrm{K}_{\mathrm{TR}}=\mathrm{K}_{\mathrm{TR}}(\underline{\mathrm{u}})=\prod_{\mathrm{i}=1}^{\mathrm{m}} \mathrm{K}_{\mathrm{i}}\left(\mathrm{u}_{\mathrm{i}}\right) \text {, }
$$

where $\mathrm{u}_{\mathrm{i}}=\mathrm{u}_{\mathrm{i}}(\gamma)$-lower confidence bounds for the parameters of individual elements with the same confidence coefficient; $\gamma, \mathrm{T}-$ is the number of LTE subsystems.

Equation (14) shows that the lower confidence bound for the $K_{T R}$ is preserved during the transition from individual elements to the LTE as a whole. Failure prevention leads to an increase of $\mathrm{K}_{\mathrm{TR}}$ and minimizes operating costs.

The impact of $\mathrm{K}_{\mathrm{TR}}$ on the cost of LUO can be estimated through a measure of efficiency, which can be represented as the $\mathrm{M}_{\mathrm{EF}}$ coefficient:

$$
\mathrm{M}_{\mathrm{EF}}=\frac{\Delta \mathrm{K}_{\mathrm{TR}}}{\Delta\left(\frac{T_{\mathrm{BP}}}{T_{\mathrm{p}}}\right)},
$$

where $\Delta K_{T R}=K_{i+1}-K_{i}-$ the increment of the technical readiness coefficient of the LTE during the transition from one technical condition $i$ to another $(i+1)$, 


$$
\Delta\left(\frac{T_{B P}}{T_{p}}\right)=\left(\frac{T_{B P}}{T_{p}}\right)_{i}-\left(\frac{T_{B P}}{T_{p}}\right)_{i+1}-\text { increment of time } \mathrm{T}_{\mathrm{BP}} \text { and } \mathrm{T}_{\mathrm{p}} \text { when changing reliability }
$$

of LTE.

This approach to determining the definition of $\mathrm{K}_{\mathrm{TR}}$ and $\mathrm{T}_{\mathrm{BP}}$ is possible only with one-time work. At StC terminals, this approach can only be applied when handling single shipments or cargo (for example, non-dimensional cargo). In the equation (13), the $\mathrm{T}_{\mathrm{BP}}$ indicator, which is subject to optimization, in particular, when reducing the downtime for emergency repairs, entails an increase in the value of $\mathrm{K}_{\mathrm{TR}}$. The equation (10) given in work [19] expresses the economic dependence of the $\mathrm{K}_{\mathrm{TR}}$ on the quantity and quality of the repair and restoration work carried out. The use of $\mathrm{K}_{\mathrm{TR}}$ shows the effectiveness of the TO strategy and takes into account the reliability of the LTE in the process of its operation.

\section{Discussion}

The considered TO strategies throughout the life cycle of the LTE depend on the adopted technical policy of the StC. The effectiveness of the functioning and management of the TO system of LTE requires further research. The existing separate studies of TO strategies are not combined into a single methodological complex, and were developed under certain operating conditions of technical objects.

The effectiveness of the chosen TO strategy can be estimated by the value of the $\mathrm{K}_{\mathrm{TR}}$, since this coefficient takes into account only the downtime of the LTE during emergency repairs. Planned MR works are not taken into account when calculating the $\mathrm{K}_{\mathrm{TR}}$, since at this moment the LTE is in working or operable condition.

When calculating the $K_{u}$, all LTE downtime is taken into account, even when routine maintenance is performed in "windows", so the $\mathrm{K}_{\mathrm{u}}$ cannot be used as an indicator of the effectiveness of the selected TO strategy.

\section{References}

1. RD 31.1.02-04. Rules for the technical operation of lifting and transport equipment in commercial seaports (CJSC "Central Research and Design Institute of the Navy", St. Petersburg, 2004)

2. I.V. Zub, Y.E. Ezhov, V.A. Sidorenko, Bulletin of the Admiral Makarov State University of Maritime and Inland Shipping 10(6), 1152-1161 (2018) DOI: 10.21821/2309-51802018-10-6-1152-1161.

3. D.V. Varnakov, V.V. Varnakov, M.E. Dezhatkin, Bulletin of the Ulyanovsk State Agricultural Academy 2(38), 168 - 173 (2017) DOI 10.18286/1816-4501-2017-2-168173

4. S.I. Makarenko, Control, communication, and security systems 1, 278-287 (2016)

5. N.N. Smirnov, A.A. Itskovich, Maintenance and repair of aircraft equipment according to the condition (Transport, M., 1987)

6. G.D. Kokorev, I.A. Uspensky, I.N. Nikolotov, Agro engineering 3, 72 - 75 (2009)

7. V.S. Kvaginidze, N.A. Koretskaya, N.N. Chupeikina, V.V. Akimenko, A.V. Akhremenkov, Mining Information and Analytical Bulletin (scientific and technical journal) S3, 310 - 314 (2011)

8. E.M. Bashirova, U.F. Yumaguzin, R.T. Yulberdin, Transportation and storage of petroleum products and hydrocarbons 1, 18-22 (2014) 
9. A.T. Martirosyan, Internet-journal "NAUKOVEDENIE" 9(6) (2017) https://naukovedenie.ru/PDF/164EVN617

10. A.L. Manakov, A.Y. Kirpichnikov, T.K. Tyunyukova, Bulletin of the Irkutsk State Technical University 5(100), 127-131 (2015)

11. O.N. Osnovina, L.M. Boeva, Mining Information and Analytical Bulletin (scientific and technical journal) 3, 251-255 (2015)

12. E.E. Bueshev, A.N. Potapov, Scientific Bulletin of the military-industrial complex of Russia 4, 80 - 86 (2016)

13. G. Medvedeva, A. Museridze, Y. Tikhonova, A. Kryukov, A. Zaydullin, SAPR (computer-aided design tools) and Graphics 1, 84-89 (2013)

14. A.I. Vinnik, N.G. Makarenko, A.M. Smirnov, A.A. Shargaev, Bulletin of the Samara Scientific Center of the Russian Academy of Sciences 18.1(2), 161 - 165 (2016)

15. V.A. Ivanov, A.A. Feshchenko, Bulletin of the Perm National Research Polytechnic University. Mechanical engineering, materials science 20(3), $82-89$ (2018) DOI: 10.15593/2224-9877/2018.3.10

16. I.I. Vainshtein, G.E. Mikhalchenko, Y.V. Vainshtein, K.V. Safonov, Bulletin of Reshetnev Siberian State University of Science and Technology 2(54), 20-25 (2014)

17. A.T. Lebedev, A.A. Seregin, A.G. Arzhenovsky, Improving the efficiency of the functioning of machines and equipment of the agro-industrial complex by managing the reliability of their systems 2(46), 4-11 (2019)

18. A.N. Dyakov, Proceedings of the Military Space Academy named after A. F. Mozhaisky 660, $180-189$ (2018)

19. I.I. Vainshtein, G.E. Mikhalchenko, Y.V. Vainshtein, K.V. Safonov, Vestnik Reshetnev Siberian State University of Science and Technology 16(3), 645-650 (2015)

20. I.V. Pavlov, S.V. Razgulyaev, Bulletin of the Bauman Moscow State Technical University. Natural sciences 4, 15-22 (2015) 